\title{
Diversidade de bromeliáceas epífitas na Área de Proteção Ambiental Ilha do Combu, Belém, Pará, Brasil
}

\author{
Adriano Costa Quaresma ${ }^{1,2}$ e Mário Augusto G. Jardim ${ }^{1}$
}

Recebido em 17/08/2011. Aceito em 12/12/2011

\begin{abstract}
RESUMO
(Diversidade de bromeliáceas epífitas na Área de Proteção Ambiental Ilha do Combu, Belém, Pará, Brasil). Informações sobre a diversidade no nível de espécie oferecem subsídios importantes para o desenvolvimento sustentável e a conservação biológica. Neste contexto, estudos com Bromeliaceae merecem destaque, especialmente porque o grupo é importante ecologicamente, mas ainda pouco conhecido na Região Norte. Neste trabalho, foram demarcadas duas parcelas de 100 m x $100 \mathrm{~m}$ em uma floresta de várzea localizada na Área de Proteção Ambiental Ilha do Combu, em Belém, Estado do Pará. Cada parcela foi subdividida em oito parcelas de $50 \mathrm{~m}$ x $50 \mathrm{~m}$, tendo todas as espécies e espécimes de bromeliáceas epífitas registradas e quantificadas. A diversidade do grupo foi calculada utilizando o índice de Shannon-Wiener. Foram registrados 1.339 indivíduos pertencentes a oito espécies e quatro gêneros. Tillandsia e Aechmea apresentaram maior riqueza. A diversidade de espécies na área foi de $\mathrm{H}=1,10$, apresentando dominância acentuada de muitos indivíduos em poucas espécies.
\end{abstract}

Palavras-chave: Amazônia, Bromeliaceae, Floresta de Várzea, Epifitismo

\begin{abstract}
(Diversity of epiphytic bromeliads in the environmental protection area of Combu Island, Belém, Pará, Brazil). Information about diversity at the species level offers data for sustainable development and biological conservation. In this context, studies about Bromeliaceae are noteworthy, especially because this group is ecologically important and poorly known in the North Region of Brazil. In this study, two grids ( $100 \mathrm{~m}$ x $100 \mathrm{~m})$ were delineated in a floodplain forest in the environmental protection area of Combu Island, Belém, Pará, Brazil. The grids were subdivided into eight grids of $50 \mathrm{~m} \mathrm{x} 50 \mathrm{~m}$, and all species and individuals of epiphytic Bromeliaceae were recorded and quantified. The diversity was calculated using the Shannon-Wiener index. A total of 1,339 individuals, belonging to eight species and four genera were recorded. Tillandsia and Aechmea presented the greatest richness. The diversity of species was $\mathrm{H}=1.10$, presenting sharp dominance of many individuals of few species.
\end{abstract}

Key words: Amazon, Bromeliaceae, Floodplain Forest, Epiphytism

\section{Introdução}

A floresta amazônica é o maior reservatório natural da diversidade vegetal do planeta, e cada ambiente florestal possui um contingente florístico rico e variado, muitas vezes endêmico (Oliveira \& Amaral 2004). O conhecimento de floras regionais e as informações da diversidade no nível de espécie oferecem subsídios importantes para o desenvolvimento sustentável e a conservação biológica (Lima \& Wanderley 2007). As epífitas perfazem cerca de 10\% da flora mundial, sendo representadas principalmente por orquídáceas, cactáceas, pteridófitas e bromeliáceas (Gentry \& Dodson 1987), e ajudam a promover um ambiente apropriado para a interação com uma grande variedade de animais e microorganismos, contribuindo para manutenção da biodiversidade (Lima \& Wanderley 2007). São plantas que estabelecem todo ou parte do seu ciclo de vida sobre troncos, galhos, ramos e folhas das árvores (forófitos), sem emissão de estruturas haustoriais como fazem as plantas parasitas (Benzing 1990).

As Bromeliaceae possuem aproximadamente 3.086 espécies e estima-se que $40 \%$ delas e $70 \%$ dos gêneros ocorram no Brasil (Wanderley et al. 2007). Foram consideradas em levantamentos amplos de epífitas vasculares (Kersten \& Siva 2001, 2002; Hefler \& Faustioni 2004; Dettke et al. 2008; Kersten \& Kunyioshi 2009; Wanderley et al. 2007; Bataghin

\footnotetext{
Museu Paraense Emílio Goeldi, Coordenação de Botânica, Belém, PA, Brasil

2 Autor para correspondência: acq.quaresma@gmail.com
} 
et al. 2010; Rogalski \& Zanin 2003; Buzatto et al. 2008), porém outros trabalhos (Alves 2005; Bonnet \& Queiroz 2006; Bonnet et al. 2007a,b; Martinelli et al. 2008; Reis \& Fontoura 2009; Fontoura \& Santos 2010) dedicaram-se à família como um todo. Para Amazônia extra brasileira, são encontrados alguns trabalhos com epífitos vasculares na Venezuela (Nieder et al. 2000; Hernández-Rosas 2001) e Colômbia (Triana-Moreno et al. 2003). Na Amazônia brasileira, destaca-se o levantamento de Bromeliaceae realizado por Ribeiro et al. (1999), em floresta de terra firme; por outro lado, os trabalhos ecológicos com epífitas de Gottsberger \& Morawetz (1993), em savana, e o de Pos \& Sleegers (2010), em floresta de várzea, não registraram bromeliáceas.

$\mathrm{Na}$ Amazônia brasileira, as bromeliáceas são encontradas com maior frequência em locais de vegetação de baixios, campina, campinarana e igapó (Sousa \& Wanderley 2007). Nas áreas de várzea, não são encontradas espécies terrestres, pois a variação no nível das águas é um fator decisivo na mortalidade do grupo (Leme \& Marigo 1993). Portanto, as bromeliáceas são exclusivamente epífitas neste ecossistema, não havendo ainda estudos sobre sua diversidade em várzeas estuarinas amazônicas. Dessa maneira, este estudo teve como objetivo contribuir para o conhecimento ecológico das bromeliáceas na Região Norte do Brasil, avaliando a diversidade da família na floresta de várzea da Área de Proteção Ambiental Ilha do Combu.

\section{Material e métodos}

A Área de Proteção Ambiental Ilha do Combu localiza-se no município de Belém, Estado do Pará, na margem esquerda do rio Guamá, e abrange uma área total de aproximadamente $15 \mathrm{~km}^{2}$. Situa-se a $48^{\circ} 25^{\prime} \mathrm{W}$ e $1^{\circ} 25^{\prime} \mathrm{S}$, cerca de $1,5 \mathrm{~km}$ ao sul de Belém. O solo da área de estudo é do tipo Glei Pouco Húmico, com alta percentagem de siltes e argila, e baixa percentagem de areia (Silva \& Sampaio 1998), sendo os solos de várzea baixa encharcados durante um período de tempo maior e os de várzea alta apenas nas marés mais altas (Setembro e Março). O clima é do tipo Am, segundo a classificação de Köppen, com média pluviométrica anual de $2.500 \mathrm{~mm}$ e temperatura média anual de $27^{\circ} \mathrm{C}$ (Jardim \& Vieira 2001).

O local de estudo situa-se em uma ilha fluvial, composta inteiramente de floresta de várzea (Floresta Ombrófila Densa Aluvial), ocupada continuamente por cipós, árvores, arbustos, lianas e epífitas. Apresenta estrutura e composição florística variada, sendo o açaizeiro a espécie silvestre mais abundante e de maior importância econômica (Rodrigues et al. 2006). As arbóreas dominantes são Carapa guianensis Aubl., Euterpe oleracea Mart., Hura crepitans L., Pseudobombax munguba (Mart. \& Zucc.) Dugand, Spondias mombin L., Symphonia globulifera L. f. e Virola surinamensis (Rol. ex Rottb.) Warb. (Jardim \& Vieira 2001; Cattanio et al. 2002).

Foram instaladas duas parcelas de $100 \mathrm{~m} \times 100 \mathrm{~m}$, subdivididas em oito parcelas de $50 \mathrm{~m} \times 50 \mathrm{~m}$, totalizando 2 ha de floresta. Uma parcela foi instalada em várzea alta, área com frequência de inundação menor, e outra em várzea baixa, área com frequência de inundação maior. Foram verificadas todas as árvores (forófitos) presentes nas parcelas e considerou-se indivíduo o agrupamento de rosetas (geneta). Os indivíduos foram visualizados com auxílio de binóculo Tasco Offshore e por meio de escaladas. A identificação foi baseada em Quaresma \& Medeiros (2009), além da comparação com exemplares depositados no Herbário João Murça Pires, do Museu Paraense Emílio Goeldi (MPEG), e consulta a especialista. Espécimes de bromeliáceas férteis foram coletados e depositados como material-testemunho no MPEG.

A diversidade de espécies foi analisada utilizando-se o índice de Shannon-Wiener: H'. Adicionalmente, foram calculados o índice de diversidade específica de Margalef $\left(D_{m g}\right)$ e o inverso do índice de Simpson (1/D). Também foi estimado o número de espécies na comunidade através do estimador Chao1 (Magurram 2004) e construído um diagrama de Whittaker para analisar a abundância das espécies.

\section{Resultados}

Foram registrados 1.339 indivíduos de bromeliáceas, pertencentes a oito espécies, quatro gêneros e duas subfamílias. Tillandsia L. e Aechmea Ruiz \& Pav. apresentaram maior riqueza, com quatro e duas espécies, respectivamente. Uma espécie de Tillandsia não foi identificada no nível de espécie (Tabela 1). Guzmania lingulata e Vriesea amazonica foram as espécies mais abundantes, representando juntas $88,5 \%$ dos indivíduos observados nos 2 ha estudados. Representada por apenas um indivíduo, Tillandsia anceps apresentou a menor abundância (Figura 1).

A diversidade de Shannon calculada foi $H^{\prime}=1,10$. A diversidade específica de Margalef foi $\mathrm{D}_{\mathrm{mg}}=2,23$ e o inverso de Simpson, $1 / \mathrm{D}=1,37$. O número estimado de espécies para a comunidade de bromeliáceas foi 11,5.

\section{Discussão}

Tillandsia e Aechmea predominaram em número de espécies nas duas parcelas. São dois dos maiores gêneros de Bromeliaceae e geralmente aparecem como os mais diversos em levantamentos florísticos de epífitas vasculares na Região Neotropical (Freiberg 1999; Kersten \& Silva 2001; Hefler \& Faustioni 2004; Alves 2005; Bonnet \& Queiroz 2006; Bonnet et al. 2007a,b; Dettke et al. 2008; Kersten et al. 2009; Reis \& Fontoura 2009).

A riqueza de espécies de bromeliáceas na área foi considerada baixa, principalmente quando comparada a levantamentos de epífitas na Mata Atlântica das Regiões Sul e Sudeste (Kersten \& Silva 2001, 2002; Gonçalves \& Waechter 2002; Rogalski \& Zanin 2003; Giongo \& Waechter 2004; Zipparro et al. 2005; Kersten \& Kunioshi 2009). 
Tabela 1. Número de indivíduos das bromeliáceas epífitas encontradas em duas parcelas de $100 \mathrm{~m}$ x $100 \mathrm{~m}$ na floresta de várzea da Área de Proteção Ambiental Ilha do Combu, município de Belém, Estado do Pará, Brasil, organizado em ordem decrescente de abundância das espécies.

\begin{tabular}{lcc}
\hline Espécies & $\mathrm{N}^{\circ}$ de indivíduos & (\%) do total \\
\hline Guzmania lingulata (L.) Mez & 685 & 51,3 \\
Vriesea amazonica (Baker) Mez & 499 & 37,2 \\
Tillandsia bulbosa Hook. & 94 & 7,1 \\
Tillandsia narthecioides C. Presl. & 29 & 2,2 \\
Aechmea mertensii (G. Mey) Schult. \& Schult. f. & 16 & 1,2 \\
Aechmea setigera Mart. ex Schult. \& Schult. f. & 11 & 0,8 \\
Tillandsia sp. & 4 & 0,2 \\
Tillandsia anceps G. Lodd. & 1 & 0,0 \\
\hline Total & 1339 & 100 \\
\hline
\end{tabular}

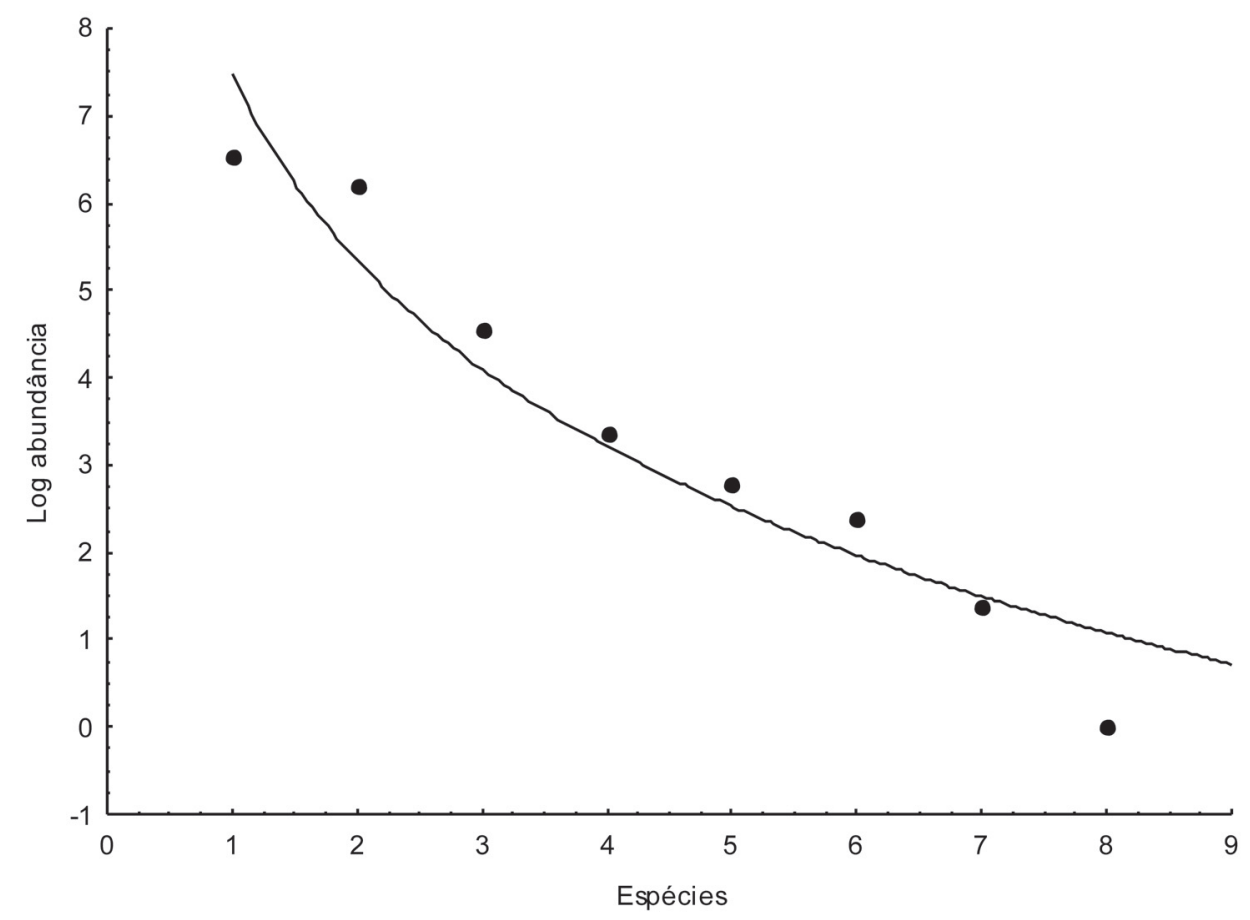

Figura 1. Diagrama de Whittaker das bromeliáceas epífitas na Área Proteção Ambiental Ilha do Combu, município de Belém, Estado do Pará, Brasil. Eixo Y: logaritmo natural das abundâncias das espécies. Eixo X: as espécies em ordem decrescente de abundância: 1. Guzmania lingulata; 2. Vriesea amazonica; 3 . Tillandsia bulbosa; 4 . Tillandsia narthecioides; 5. Aechmea mertensii; 6. Aechmea setigera; 7. Tillandsia sp.; 8. Tillandsia anceps.

Trabalhos apenas com bromeliáceas também registraram um maior número de espécies naquela região (Alves 2005; Bonnet \& Queiroz 2006; Bonnet et al. 2007a,b; Fontoura \& Santos 2010). Essa diferença era esperada, uma vez que a Mata Atlântica do Sul e Sudeste constitui um dos principais centros de diversidade da família (Wanderley et al. 2007). Entretanto, alguns levantamentos em formações ribeirinhas naquela região registraram menos espécies de Bromeliaceae do que no presente estudo (e.g., Kersten et al. 2009; Bonnet et al. 2010).

A riqueza encontrada aqui foi menor do que aquela obtida por Ribeiro et al. (1999) em uma floresta de terra firme na Amazônia central (13 espécies), porém maior que a encontrada por Nieder (2000) em uma floresta de várzea na Amazônia Venezuelana (2 espécies). Esta diferença na riqueza de espécies reflete, provavelmente, diferenças ambientais. As florestas de terra firme possuem um componente arbóreo mais rico se comparadas às florestas de várzea (Gama et al. 2005). Como as epífitas dependem das árvores para se estabelecerem, a riqueza deste componente determinará a diversidade de substratos disponíveis, influenciando a riqueza de bromeliáceas epífitas.

Guzmania lingulata e Vriesea amazonica foram as espécies de bromeliáceas mais frequentes, somando $88,5 \%$ dos 
registros. Este padrão é congruente com aquele encontrado para as bromeliáceas epífitas em plantações monoespecíficas da floresta de várzea na Costa Rica, onde Guzmania monostachya (L.) Rusby ex. Mez. e Vriesea gladioliflora (H. Wendl.) Antoine se mostraram predominantes, totalizando $98 \%$ dos indivíduos (Merwin et al. 2003). Portanto, este ecossistema parece ser caracterizado por uma elevada abundância de indivíduos em poucas espécies. A dispersão das sementes é um fator importante no estabelecimento e expansão das populações de bromeliáceas epífitas no interior da floresta (Cascante-Marín et al. 2009). Essas espécies pertencem à subfamília Tillandsioideae, possuindo sementes plumosas (Smith \& Downs 1997), o que facilita uma dispersão mais ampla, possibilitando a colonização e o seu estabelecimento em mais habitats. Apesar de Tillandsia anceps ter sido uma das bromeliáceas mais abundantes em outros estudos (Freiberg 1999; Laube \& Zotz 2006; Zotz \& Schultz 2007), apenas um exemplar foi registrado em nosso estudo. Este resultado deve estar relacionado à sua distribuição dispersa no ambiente, podendo tratar-se de uma espécie rara na APA. Outro aspecto a ser considerado é a retirada de indivíduos dessa espécie por moradores para fins paisagísticos, fato presenciado mais de uma vez durante o desenvolvimento deste trabalho.

A diversidade de espécies na floresta de várzea foi menor quando comparada com uma Floresta Estacional Semidecidual no Estado da Bahia ( $\mathrm{H}^{\prime}=2,2$; Reis \& Fontoura 2009). Os índices de Margalef e o inverso de Simpson são resultados adicionais, pois alguns autores (e.g., Magurran 2004) desaconselham o emprego do índice de Shannon. Todavia, esses índices não foram apresentados em outros trabalhos com bromeliáceas. O número de espécies encontradas ficou abaixo daquele estimado para a área, sugerindo que um maior esforço amostral poderá representar melhor a quantidade de espécies dessa comunidade.

Os estudos em Bromeliaceae na Amazônia estão restritos a levantamentos florísticos. Não existem estudos analisando a diversidade da família na região, o que dificulta avaliações comparativas. Portanto, este trabalho apresenta dados relevantes para as bromeliáceas epífitas na Amazônia, ressaltando a necessidade de mais estudos no grupo, como autoecologia de suas espécies, análises fitossociológicas e estudos ecológicos com ênfase na relação planta-animal.

\section{Agradecimentos}

Ao $\mathrm{CNPq}$, pelo apoio financeiro ao projeto "Padrões de diversidade florística, de regeneração natural e do potencial aromático em duas unidades de conservação do estado do Pará como subsídios ao plano de gestão ambiental", edital Universal-MCT/CNPq 15/2007- Processo 472260/2007-3. Ao naturalista João Batista, pela ajuda na identificação das espécies, e ao Me. Tonny David S. Medeiros, pela ajuda em campo e na discussão dos dados.

\section{Referências bibliográficas}

Alves, T.F. 2005. Distribuição geográfica, forófitos e espécies de bromélias epífitas nas matas e plantações de cacau na região de UNA, Bahia. Tese de Doutorado, Universidade Estadual de Campinas, Capinas.

Bataghin, F.A.; Barros, F. \& Pires, J.S.R. 2010. Distribuição da comunidade de epífitas vasculares em sítios sob diferentes graus de perturbação na Floresta Nacional de Ipanema, São Paulo, Brasil. Revista Brasileira de Botânica 33(3): 501-512.

Benzing, D.H. 1990. Vascular epiphytes. Cambridge, Cambridge University Press.

Bonnet, A. \& Queiroz, M.H. 2006. Estratificação vertical de bromélias epifíticas em diferentes estádios sucessionais da Floresta Ombrófila Densa, Ilha de Santa Catarina, Brasil. Revista Brasileira de Botânica 29(2): 217-228

Bonnet, A.; Curcio, G.R.; Barddal, M.L. \& Roderjan, V.R. 2007a. Estratificação vertical de bromélias epifíticas na planície do rio Iguaçu, Paraná, Brasil. Revista Brasileira de Biociências 5(1): 492-494.

Bonnet, A.; Curcio, G.R.; Barddal, M.L.; Roderjan, V.R. \& Lavoranti, J.O. 2007b. Distribuição horizontal de bromélias epífitas na planície do rio Iguaçu, Paraná, Brasil. Revista Brasileira de Biociências 5(1): 513-515.

Bonnet, A.; Curcio, G.R.; Galvão, F. \& Kozera, C. 2010. Diversidade e distribuição espacial de bromeliáceas epifíticas do altíssimo rio Tibagi - Paraná - Brasil. Floresta 40(1): 71-80.

Buzatto, C.R.; Severo, B.M.A. \& Waechter, J.L. 2008. Composição florística e distribuição ecológica de epífitos vasculares na Floresta Nacional de Passo Fundo, Rio Grande do Sul. Iheringia, série Botânica 63(2): 231-239.

Cascante-Marín, A.; Von Meijenfeldt, N.; de Leeuw, H.M.H.; Wolf, J.H.D. Oostermeijer, J.G.B. \& Den Nijs, J.C.M. 2009. Dispersal limitation in epiphytic bromeliad communities in a Costa Rican fragmented montane landscape. Journal of Tropical Ecology 25: 63-73.

Cattanio, J.H.; Anderson, A.B. \& Carvalho, M.S. 2002. Floristic composition and topographic variation in a tidal floodplain forest in the Amazon Estuary. Revista Brasileira de Botânica 25(4): 419-430.

Dettke, G.A.; Orfrini, A.C. \& Milaneze-Gutierre, M.A. 2008. Composição florística e distribuição de epífitas vasculares em um remanescente alterado de Floresta Estacional Semidecidual no Paraná, Brasil. Rodriguésia 59(4): 859-872.

Fontoura, T. \& Santos, F.A.M. 2010. Distribuição geográfica de bromélias epífitas na região de Una, nordeste do Brasil. Biota Neotropica 10(4): 127-132.

Freiberg, M. 1999. The vascular epiphytes on a Virola machellii (Myristicaceae) in French Guiana. Ecotropica 5: 75-81.

Gama, J.R.V.; Souza, A.L.; Martins, S.V. \& Souza, D.R. 2005. Comparação entre florestas de várzea e de terra firme do Estado do Pará. Revista Árvore 29(4): 607-616.

Gentry, A. \& Dodson, C.H. 1987. Diversity and biogeography of neotropical vascular epiphytes. Annals of the Missouri Botanical Garden 74: 205-233.

Giongo C. \& Waechter, J. L. 2004. Composição florística e estrutura comunitária de epífitos vasculares em uma floresta de galeria na Depressão Central do Rio Grande do Sul. Revista Brasileira de Botânica 27(3): 563-57.

Gonçalves, C.N. \& Waechter, J.L. 2002. Epífitos vasculares sobre espécimes de Ficus organensis isoladas no norte da planície costeira do Rio Grande do Sul: padrões de abundância e distribuição. Acta Botanica Brasilica 16(4): 429-441

Gottsberger, G. \& Morawetz, W. 1993. Development and distribution of the epiphytic flora in an Amazonian savanna in Brazil. Flora 188: 145-151.

Hefler, S.M. \& Faustioni, P. 2004. Levantamento florístico de epífitos vasculares no Bosque São Cristovão - Curitiba - Paraná - Brasil. Revista Estudos de Biologia 26(54): 11-19.

Hernández-Rosas, J. 2001. Ocupación de los portadores por epifitas vasculares en un bosque húmedo tropical del Alto Orinoco, Edo. Amazonas, Venezuela. Acta Científica Venezolana 52: 292-303.

Jardim, M.A.G. \& Vieira, I.C.G. 2001. Composição florística e estrutura de uma floresta de várzea do estuário Amazônico, Ilha do Combu, 
Estado do Pará, Brasil. Boletim do Museu Paraense Emílio Goeldi. Botânica 17(2): 333-354.

Kersten, R.A. \& Kuniyoshi, Y.S. 2009. Conservação das florestas na bacia do alto Iguaçu, Paraná - Avaliação da comunidade de epífitos vasculares em diferentes estágios serais. Floresta 39(1): 51-66.

Kersten, R.A. \& Silva, S.M. 2001. Composição florística e estrutura do componente epifítico vascular em floresta da planície litorânea na Ilha do Mel, Paraná, Brasil. Revista Brasileira de Botânica 24(2): 213-226.

Kersten, R.A. \& Silva, S.M. 2002. Florística e estrutura do componente epifítico vascular em Floresta Ombrófila Mista Aluvial do rio Barigui, Paraná, Brasil. Revista Brasileira de Botânica 25(3): 259-267.

Kersten, R.A.; Kuniyoshi, Y.S. \& Roderjan, C.V. 2009. Epífitas vasculares em duas formações ribeirinhas adjacentes na planície do rio Iguaçu terceiro planalto paranaense. Iheringia, série Botânica 64(1): 33-43.

Laube, S. \& Zotz, G. 2006. Long-term changes in the epiphyte vegetation of the palm, Socratea exorrhiza. Journal of Vegetation Science 17: 307-314.

Leme, E.M.C. \& Marigo, L.C. 1993. Bromélias na natureza. Rio de Janeiro, Marego comunicação visual.

Lima, T.T. \& Wanderley, M.G.L. 2007. Diversidade de Bromeliaceae da serra do Lopo Extrema - Minas Gerais. Revista Brasileira de Biociências 5(2): 1146-1148.

Magurran, A.E. 2004. Measuring biological diversity. Oxorford, WileyBlackwell.

Martinelli, G.; Vieira, C.M; Gonzalez, M.; Leitman, P.; Piratininga, A.; Costa, A.F. \& Forzza, R.C. 2008. Bromeliaceae da Mata Atlântica brasileira: lista de espécies, distribuição e conservação. Rodriguésia 59(1): 209-258

Merwin, M.C.; Rentmeester, S.A. \& Nadkarni, N.M. 2003. The influence of host tree species on the distribution of epiphytic bromeliads in experimental monospecific plantations, La selva, Costa Rica. Biotropica 35(1): 37-47.

Nieder, J.; Engwald, S.; Klawun, M. \& Barthlott, W. 2000. Spatial distribution of vascular epiphytes (including hemiepiphytes) in a lowland Amazonian rain forest (Surumoni crane plot) of southern Venezuela. Biotropica 32(3): 385-396.

Oliveira, A.N. \& Amaral, I.L. 2004. Florística e fitossociologia de uma floresta de vertente na Amazônia Central, Amazonas, Brasil. Acta Amazonica 34(1): 21-34.

Pos, E.T. \& Sleegers, A.D.M. 2010. Vertical distribution and ecology of vascular epiphytes in a lowland tropical rain forest of Brazil. Boletim do Museu Paraense Emílio Goeldi. Ciências Naturais 3(5): 335-344
Quaresma, A.C. \& Medeiros, T.D.S. 2009. As bromélias. Pp.71-77 In M.A.G. Jardim (org.), Diversidade biológica das Áreas de Proteção Ambiental Ilhas do Combu e Algodoal-Maiandeua, Pará, Brasil. Belém, MPEG/MCT/CNPq (Coleção Adolpho Ducke).

Reis, J.R.M. \& Fontoura, T. 2009. Diversidade de bromélias epífitas na Reserva Particular do Patrimônio Natural Serra do Teimoso - Jussari, BA. Biota Neotropica 9(1): 73-79.

Ribeiro, J. E. L. S.; Hopkins, M.J.G; Vicentini, A.; Sothers, C.A.; Costa M.A.S.; Brito, J.M.; Souza, M.A.D.; Martins, L.H.P.; Lohmann, L.G.; Assunção, P.A.C.L.; Pereira, E.C.; Silva, C.F.; Mesquita, M.R. \& Procópio, L.C. 1999. Flora da Reserva Ducke: guia de identificação das plantas vasculares de uma floresta de terra-firme na Amazônia Central. Manaus, Instituto Nacional de Pesquisas da Amazônia.

Rodrigues, L.M.B.; Lira, A.U.S.; Santos, F.A. \& Jardim, M.A.G. 2006 Composição florística e usos das espécies vegetais de dois ambientes de floresta de várzea. Revista Brasileira de Farmácia 87(2): 45-48.

Rogalski, J.M. \& Zanin, E.M. 2003. Composição florística de epífitos vasculares no estreito de Augusto César, Floresta Estacional Decidual do Rio Uruguai, RS, Brasil. Revista Brasileira de Botânica, 26(4): 551-556.

Silva, C.A.R. \& Sampaio, L.S. 1998. Speciation of phosphorus in a tidal floodplain forest in the Amazon Estuary. Mangrove and Salt Marshes 2(1): 51-57.

Smith, L. B. \& Downs, R.J. 1977. Tillandsioideae (Bromeliaceae). Flora Neotropica 14(2): 663-1492.

Sousa, G.M \& Wanderley, M.G.L. 2007. Aechmea rodriguesiana (L.B. Sm) L.B. Sm. (Bromeliaceae) uma espécie endêmica da Amazônia. Acta Amazonica 37(4): 517-520.

Triana-Moreno, L.A.; Garzón-Venegas, N.J.; Sánchez-Zambrano, J. \& Vargas, O. 2003. Epífitas vasculares como indicadores de regeneración en bosques intervenidos de la Amazonía Colombiana. Acta Biológica Colombiana 8: 31-42.

Wanderley, M.G.L. \& Martins, S.E. 2007. Bromeliaceae. Pp. 39-161 In: M.G.L. Wanderley, G.J. Shepherd, T.S. Melhem \& A.M. Giulietti. Flora fanerogâmica do Estado de São Paulo, Vol. 5. São Paulo, Instituto de Botânica.

Zipparro, V.B.; Guilherme, F.A.G.; Almeida-Scabbia, R.J. \& Morellato, L.P.C. 2005. Levantamento florístico de floresta atlântica no sul do Estado de São Paulo, Parque Estadual Intervales, base Saibadela. Biota Neotropica 5(1): 147-170.

Zotz, G. \& Schultz, S. 2007. The vascular epiphytes of a lowland forest in Panama - species composition and spatial structure. Plant Ecology 195: 131-141. 